\title{
Percutaneous Screw Fixation of Distal Tibia Fractures Functional Results in Sixteen Patients
}

\author{
Igor Kaftandziev, Simon Trpeski, Oliver Arsovski, Marko Spasov* \\ University Clinic of Traumatology, Faculty of Medicine, Ss Cyril and Methodius University of Skopje, Skopje, Republic of \\ Macedonia
}

\author{
Citation: Kaftandziev I, Trpeski S, Arsovski O, \\ Spasov M. Percutaneous Screw Fixation of \\ Distal Tibia Fractures - Functional Results in \\ Sixteen Patients. OA Maced J Med Sci. 2014 \\ Jun 15; 2(2):229-233. \\ http://dx.doi.org/10.3889/oamjms.2014.036 \\ Key words: tibia; distal; fracture; percutaneous; \\ outcome. \\ "Correspondence: Dr. Marko Spasov. \\ University Clinic of Traumatology, Emergency \\ Department, Faculty of Medicine, Ss Cyril and \\ Methodius University of Skopje, Vodnjanska 17, \\ Skopje 1000, Republic of Macedonia. Phone: \\ 071607956. E-mail: marspasov@yahoo.com \\ Received: 29-Dec-2013; Revised: 08-Mar- \\ 2014; Accepted: 30-Mar-2014; Online first: \\ 04-Apr-2014 \\ Copyright: (c) 2014 Kaftandziev et al. This is \\ an open-access article distributed under the \\ terms of the Creative Commons Attribution \\ License, which permits unrestricted use, \\ provided the original author and source are \\ provided
credited. \\ Competing Interests: The authors have \\ declared that no competing interests exist.
}

\begin{abstract}
INTRODUCTION: An important feature of distal tibia fractures is the relevance of the soft tissue coverage. In order to maintain good functional outcome, several operative techniques have been established. Among them, percutaneous screw fixation has the advantage of causing less biological damage of the soft tissues with lower rates of complications.
\end{abstract}

MATERIAL AND METHODS: We reviewed 16 patients with distal tibia fracture. Operative treatment consisted of indirect reduction and percutaneous fixation of the tibia followed by cast immobilization for approximately 3-4 months. Minimum follow up period was 12 months. Functional outcome was evaluated using Olerud-Molander Ankle Score (OMAS) and American Orthopaedic Foot and Ankle Society ankle-hindfoot score (AOFAS).

RESULTS: Twelve out of 16 fractures healed within 6 months of the injury. In 3 cases, delayed union was evident, but the fractures healed in less than nine months. There was one case of malunion, two cases of superficial skin infections, two cases of DVT and two cases of gross swelling. According to OMAS score, the functional result was excellent in $7(43.75 \%)$, good in 5 $(31.25 \%)$ and fair in $4(25 \%)$ patients.

CONCLUSION: Percutaneous screw fixation can be a safe and effective method for operative treatment of distal tibia fractures.

\section{Introduction}

Fractures of the distal tibia are one of the most challenging injuries in orthopedic surgery. They involve the diaphyseal-metaphyseal area of the bone and may be either extra-articular or intra-articular, the latter known as pilon or plafond fractures. These are considered severe injuries because of the elevated risk of complications which may result from the nature of injury itself, secondary to surgery or both [1-3].

During the past decades, four basic principles of treatment were established and their strict application was considered to be essential in order to obtain good result. These included reconstruction of fracture of the fibula, restitution of articular surface of the tibia, autologous bone graft and medial buttress of the tibia.

In order to maintain reduction, several operative techniques have been advocated. The development of new implants and techniques has helped considerably in obtaining stable fixation in these fractures. Distal metaphyseal fractures without or with simple articular involvement are candidates for intramedullary nailing. On the other hand, pilon fractures with extensive articular involvement are almost impossible to treat by closed reduction and percutaneous fixation. Despite of all this progress in the operative techniques, the clinical results are not always successful and it is quite difficult to predict the final outcome [4-6].

An important characteristic of fractures of distal tibia, particularly those caused by high energy is that the bony injury cannot be separated from the injury of the surrounding soft tissues [7, 8].

According to $\mathrm{H}$. Tscherne, "soft tissue injuries accompanying closed fractures are especially troublesome and are often insufficiently appreciated on account of their occult nature". Even a simple skin contusion over a closed fracture can pose a more complex range of therapeutic and prognostic problems than skin which has been broken by fractured bone [9]. Therefore, the operative strategy used in dealing with distal tibia fractures is frequently much influenced by the soft tissue condition. Timing of 
surgery, appropriate operative strategies and delicate atraumatic handling with the soft tissues are of vital importance for the management of these fractures in order to achieve good postoperative results and to minimize the possible complications.

Percutaneous fracture fixation of distal tibia fractures has been shown to be a successful technique with minimal complications. The main advantage of percutaneous fixation is minimizing biologic damage to soft tissues, and decreasing of the rates of infection and nonunion [10-12].

The aim of this study was to determine the clinical and functional outcome after distal tibia fracture in patients treated operatively with indirect reduction and percutaneous fixation in our institution.

\section{Material and Methods}

In this study, the clinical material from our institution was used. We reviewed 16 patients (12 male, 4 female) between January 2009 and December 2011.

The mechanism of injury was high energy trauma in 10 patients (traffic accident) and in 6 patients, fall from height. The mean age of the patients was 34 years (range 19-56 years).

The examinations were performed following the determined criteria. The same preoperative, operative and postoperative protocol was conducted in all patients. Minimum follow-up was 12 months. Power calculation was not undertaken.

The preoperative protocol consisted of clinical evaluation of the general condition of the patient and evaluation of the injury, especially the soft tissue damage. Standard laboratory and radiological investigations (AP and profile standard radiographs) were performed immediately after the injury, after fracture repair and at the regular check up.

The inclusion and exclusion criteria for the study were defined. Extra-articular metaphyseal fractures and partial articular pilon fractures where a part of the articular surface remains in contact with the shaft and without articular surface impaction were included in the examination. Diaphyseal fractures of the tibia, complete articular pilon fractures where no part of the articular surface remains in contact with the shaft and open fractures were excluded.

We used AO classification system of fractures and Tscherne and Oestern classification of injuries of the soft tissues $[13,14]$. Using the $A O$ classification, 8 fractures were type 43- A (three group A1, four group $A 2$ and one group A3), 3 fractures were type 43- B (one group B1, two group B2). All type 43-C fractures were group $\mathrm{C} 1$. According to Tscherne and Oestern classification of soft tissue injuries with closed fractures, ten, five and one patients were graded type
0,1 and 2 respectively.

All the patients were operatively treated within 8 hours of the injury. Patients were positioned supine on a radiolucent table. Fractures were reduced under fluoroscopy. The reduction was achieved indirectly, with slight traction and percutaneous reduction with clamps. The Indirect fracture reduction technique was used to avoid disruption of the fracture environment and to minimize the soft tissue additional damage. The fibula was typically stabilized first with open reduction and internal fixation to improve stability and aid in tibia reduction. Definitive stabilization of the distal tibia fracture was performed using percutaneous canulated screws.

Antero-posterior and lateral radiographs were taken to assess fracture reduction. In all the patients, plaster splint was applied for ten days. Antibiotic therapy (third generation cephalosporin) for 5-7 days was administrated. Low-molecular weight heparin (40 mg subcutaneously) was administrated in a period of six weeks post injury. Early postoperative evaluation of the soft tissue condition was done.

According to the postoperative protocol, after wound healing and removal of the stitches, cast was applied and patients were not allowed to weight bear for 4 to 6 weeks. In the following 4 weeks only partial weight bearing (maximum $25 \mathrm{~kg}$ ) was allowed. Full weight bearing was initiated when early clinical and radiographic signs of healing were present (3 or 4 months after the operation).

All patients followed medical physiotherapy and rehabilitation process.

The patients were followed up until clinical and radiologic union occurred. Standard follow up was conducted every 6-8 weeks with control clinical and radiological exams, for a minimum 12 months and longer, if any associated complications appeared.

Several parameters, such as: radiological outcome with a point of the time of union and problems of union, soft tissue condition, other complications related to the operative treatment and the functional outcome were also evaluated.

Clinical outcomes were assessed on the final follow up at 12 months post injury.

The radiological outcome was evaluated following the determined criteria. Fracture healing within the first 6 months was defined as normal, from 6 to 9 months as delayed and more than 9 months as nonunion. The fracture was considered healed when patient was fully weight bearing without pain or significant local discomfort and radiographs demonstrated at least three cortices of bony bridging. Malalignment was defined as more than 5 degrees angulation in any plane or shortening more than $1 \mathrm{~cm}$.

Outcome was measured using standard questionnaires, and consisted of the American 
Orthopaedic Foot and Ankle Society ankle-hindfoot score (AOFAS) and Olerud-Molander Ankle Score (OMAS). The AOFAS ankle hindfoot score consists of nine questions related to three components: pain (one question; 10 points, function (seven questions; 50 points) and alignment (one question; 10 points) with total possible score of 100 points [15]. The question related to the alignment and range of motion was completed by a surgeon based upon radiographs. Other questions were completed by the patient. OMAS score [16] includes nine different items with a score of zero (totally impaired) to 100 (completely unimpaired). According to this score, results were considered as excellent (>92 points), good (92-87 points), fair (86-65 points) and poor (<65 points).

\section{Results}

All of 16 approached patients in our study answered the questionnaire. Table 1 shows the baseline characteristics of the patients.

Table 1: Patient Characteristics.

\begin{tabular}{|c|c|c|c|c|c|c|c|}
\hline Patient & Sex & Age & AO & T-O & $\begin{array}{c}\text { Time of } \\
\text { union/ weeks }\end{array}$ & Compl. & $\begin{array}{l}\text { OMAS } \\
\text { Score }\end{array}$ \\
\hline 1 & $\bar{M}$ & 26 & $\overline{B 1}$ & 0 & 24 & - & 95 \\
\hline 2 & $M$ & 41 & $\mathrm{~A} 1$ & 0 & 20 & - & 97 \\
\hline 3 & M & 45 & $\mathrm{C} 1$ & 1 & 30 & - & 80 \\
\hline 4 & $\mathrm{~F}$ & 28 & $\mathrm{C} 1$ & 0 & 23 & DVT & 89 \\
\hline 5 & M & 19 & A2 & 0 & 22 & - & 98 \\
\hline 6 & $M$ & 40 & A3 & 1 & 19 & INF & 89 \\
\hline 7 & M & 31 & B2 & 1 & 26 & - & 79 \\
\hline 8 & $\mathrm{~F}$ & 51 & $\mathrm{C} 1$ & 2 & nonunion & INF & 93 \\
\hline 9 & $\mathrm{~F}$ & 37 & $\mathrm{C} 1$ & 0 & 26 & GS & 95 \\
\hline 10 & M & 45 & A2 & 0 & 31 & - & 94 \\
\hline 11 & $M$ & 56 & $\mathrm{C} 1$ & 1 & 33 & - & 88 \\
\hline 12 & M & 33 & $\mathrm{~A} 1$ & 0 & 25 & malunion & 82 \\
\hline 13 & M & 23 & A2 & 0 & 21 & - & 80 \\
\hline 14 & M & 21 & B2 & 1 & 23 & GS & 89 \\
\hline 15 & M & 28 & A2 & 0 & 23 & - & 90 \\
\hline 16 & $\mathrm{~F}$ & 34 & A1 & 0 & 24 & DVT & 93 \\
\hline
\end{tabular}

M - Male; F - Female; AO - AO Classification; T-O - Tscherne-Oestern
Classification; GS - Gross Swelling; DVT - Deep Vein Thrombosis; INF - Infection; Compl. - Complication

Most of the patients were male and the median age at trauma was 34 years. Radiological outcome was based on a regular follow up conducted every 6-8 weeks, for a minimum of 12 months (Fig.1). Follow up results showed that in 12 patients, fracture healed within 6 months of the injury. The average time of union was 23 weeks (range 19-26 weeks). Of these 16 fractures, three fractures however had delayed union, with union time of less than nine months. In one patient, nonunion was evident 9 months after the injury.

Despite anatomical reduction, one patient developed sterile 15 degrees valgus malunion. The result of OMAS score in this patient was fair (82 points), and the patient predominantly had difficulties while running and jumping.
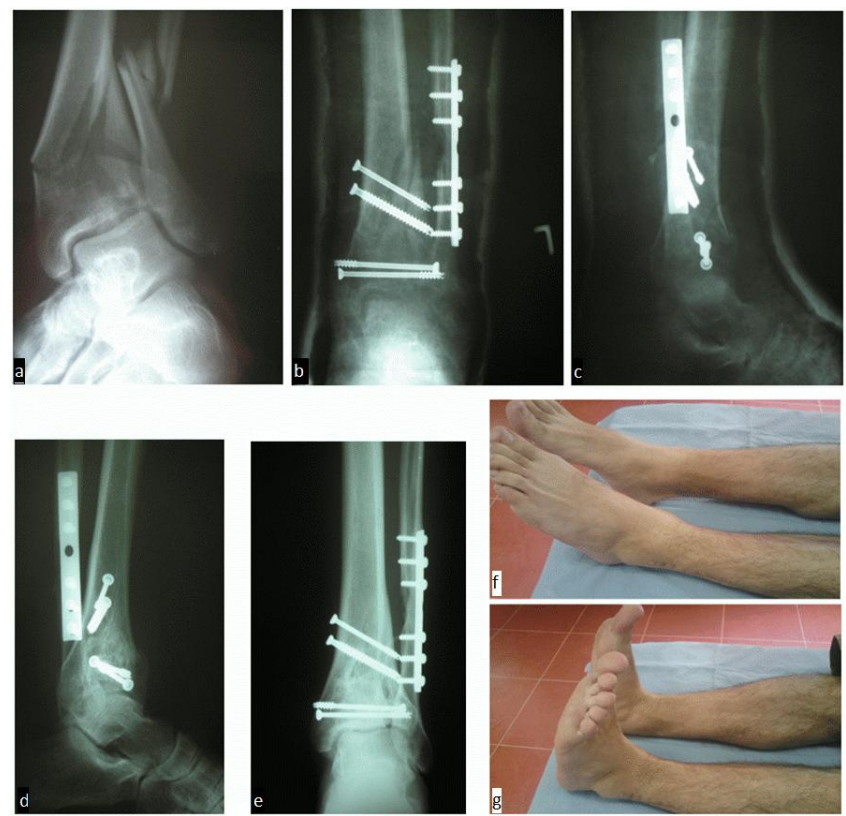

Figure 1: a - Preoperative; b, c - Early postoperative; $d$, e - 6 months after injury; $\mathrm{f}, \mathrm{g}$ - range of motion, 6 months after injury.

Superficial wound infection was observed in 2 patients with Tscherne group 1 and 2 closed injury. Stitches were removed and wounds healed secondarily with topical care. There were two cases of DVT and two cases of persistent swelling.

The average value according to OMAS score was 88 . In $7(43.75 \%)$ patients the result was excellent, in $5(31.25 \%)$ good and in $4(25 \%)$ fair. The average value of AOFAS score was 85 (range 65100).

\section{Discussion}

The results of surgical treatment of distal tibia fractures continue to be somewhat disappointing. These are fractures which commonly need subsequent surgery. Pain sufficient to daily activities is notified in most of the patients and restriction of ankle motion is almost certain following this injury.

Perfect anatomical reconstruction is still considered the most important goal in articular fractures. Early surgery accomplished with a traumatic dissection, resulting in anatomic reduction secured by rigid fixation and followed by early active motion and delayed weight bearing gives the chance of successful outcome $[12,17]$.

Distal tibia fractures have been particularly problematic because of the sparse soft tissue coverage and relatively poor blood supply in this location with a thin layer of skin and subcutaneous tissue over the anteromedial surface of the bone [18, 19]. 
Borelli showed that the risk of disruption the blood supply in the metaphysical region of the tibia is increased with classical open reduction and internal fixation technique. This led to development of MIPO operative technique. Percutaneous plate osteosynthesis of distal tibia metaphyseal fractures is a feasible and worthwhile alternative method of stabilization, avoiding some of the soft tissue complications associated with conventional open plate technique. Over the last decade, minimally-invasive plate osteosynthesis using indirect fracture reduction has been successful in treating complex fractures of the lower extremity. The goal of this technique is to apply a stable plate fixation, maintain the fracture biology and respect the soft tissues [11, 12, 20].

Nowadays the development of anatomicallycontoured plates, along with angular stability and distal locking options with IM nails has helped considerably in obtaining stable fixation in these difficult fractures.

In a context of minimally invasive approach to this type of fractures, the usage of stabilization with percutaneus canulated screws should be emphasized. Although literature data for this type of fixation are lacking, our results were similar to the published data of the MIPO techniques studies. According to our experience, the main problem of performing this technique of fixation is the maintaining of stable osteosynthesis or keeping the stability after osteosyntesis. Therefore, the use of cast is necessary, although the long term immobilization, followed by discomfort because of the cast and disability for early joint motion are considered as disadvantages. After the removal of the cast, a prolonged period of rehabilitation is required for obtaining good final functional results. The performance of this operative technique is connected to the use of fluoroscopy. On the other hand, the damage of soft tissue is minimal. According our experience, the best time to perform the operative technique is in the first hours after the trauma. Therefore, the clinical evaluation of the soft tissue condition and the radiological evaluation of the fracture type are crucial for the performance of the procedure. Our results showed that good final functional results and low percentage of complication could be obtained in patients with fracture type $A 1$, A2, A3, type B1 and B2 and type C1, compared to studies of MIPO operative technique of the fracture fixation. In fractures with significant articular involvement (group C2 and C3), it is almost impossible to achieve anatomical reduction and stable fixation by using the presented technique.

Limitations of this study are low number of patients, uncohort group of patients, short follow up period and having no alternative treatment or a control group for comparison of the results.

In conclusion, the key point in this operative technique is the preoperative planning. It should include the optimal timing of surgery, considering further impairment of critical soft tissue during the posttraumatic course.

Unfortunately, even an excellent pre-operative plan, along with careful attention to the soft tissues and well performed surgery may still result in poor outcome due to primary articular cartilage damage at the time of the injury.

Percutaneous screw fixation can be a safe and effective alternative method for operative treatment of distal tibia fractures. This operative technique enables low percentage of complications and good final outcome. The main disadvantages are the discomfort because of the cast and inability for early joint motion which requires prolonged period of rehabilitation.

\section{References}

1. Hahn MP, Thies JW. Pilon tibiale fractures. Chirug. 2004 75:211-30.

2. Assal M, Ray A, Stern R. The extensile approach for the operative treatment of high-energy pilon fractures: surgical technique and soft tissue healing. J Orthop Trauma. 2007; 21 196-206.

3. Pollak $\mathrm{AN}$ et al. Outcomes after treatment of high energy tibia plafond fractures. J Bone Joint Surg Am. 2003; 1893-900.

4. Borg T, Larsson S, Lindsjo U. Percutaneous plating of distal tibia fractures. Preliminary results in 21 patients. Injury. 2004; 35:608-614.

5. Lau TW, Leung F, Chan CF, et al. Wound complication of minimally invasive plate osteosyntesis in distal tibia fractures. Int Orthop. 2008; 32:697- 703.

6. Helfet DL, Shonnard RY, Levine D, et al. Minimally invasive plate osteosyntesis of distal fractures of the tibia. Injury. 1997; 28 (Suppl 1): S- A42- S- A48.

7. Redfern DJ, Syed SU, Davies SJM. Fractures of the distal tibia: minimally invasive plate osteosyntesis. Injury. 2004; 35 : 615- 620 .

8. Hasenboehler E, Rikli D, Babst R. Locking compression plate with minimally invasive plate osteosyntesis in diaphyseal and distal tibial fracture: a retrospective study of 32 patients. Injury. 2007; 38: 365- 370.

9. H. Tscherne J Schatzker: Major Fractures of the Pylon, the Talus and the Calcaneus; Current Concepts of Treatment Springer-Verlag, Berlin 1993.

10. Collinge $\mathrm{C}$, Sanders $\mathrm{R}$, DiPasquale $\mathrm{T}$. Treatment of complex tibial periarticular fractures using percutaneous techniques. Clin Orthop Relat Res. 2000; 375:69- 77.

11. Borrelli J Jr, Prickett W, Song E, et al. Extraosseous blood supply of the tibia and the effects of different plating techniques: a human cadaveric study. J Orthop Trauma. 2002; 16:691-695.

12. Krackhardt T, Dilger J, Flesch I, et al. Fractures of the distal tibia treated with closed reduction and minimally invasive plating. Arch Orthop Trauma Surg. 2005; 125:87-94

13. Muller $M$, Nazarian $S$, Koch $P$, Schatzker J. The Comprehensive Classification of Fractures of long Bones. Edited, Springer: Berlin, 1990

14. Tscherne $\mathrm{H}$, Oestern $\mathrm{H}$. Pathophysiology and classification of soft tissue injuries associated with fractures. In: Tscherne $H$, 
Gotzen L, eds. Fractures with Soft Tssue Injuries; Springer: Berlin, 1984: 1-9.

15. Kitoaka HB, Alexander IJ, Adelaar RS, Nunley JA, Myerson MS, Sanders M. Clinical rating systems for the ankle-hindfoot, midfoot, hallux and lesser toes. Foot Ankle Int. 1994; 15(7):349-353.

16. Olerud $\mathrm{C}$, Molander $\mathrm{H}$. A scoring scale for symptom evaluation after ankle fracture. Arch Orthop Trauma Surg. 1984; 103(3): 190-194.

17. Bilat C, Leutenegger A, Ruedi T. Osteosyntesis of 245 tibial shaft fractures: early and late complications. Injury. 2004; 35:349-358

18. Konrath G, Moed BR, Watson T, et al. Intramedullary nailing of unstable diaphyseal fractures of the tibia with distal intraarticular involvement. J Orthop Trauma. 1997; 11:200205.

19. Obremskey WT, Medina M. Comparison of intramedullary nailing of distal third tibial shaft fractures: before and after traumatologists. Orthopedics. 2004;27:1180- 1184.

20. Maffulli N, Toms AD, McMurtie A, Oliva F. Percutaneous plating of distal tibial fractures. Int Orthop. 2004; 28: 159- 162.

21. Janssen KW, Biert J, van Kampen A. Treatment of distal tibial fractures: plate versus nail. A retrospective outcome analysis of matched pairs of patients. Int Orthop. 2007; 31:709-714.

22. Vallier HA, Le TT, Bedi A. Radiographic and clinical comparisons of distal tibia shaft fractures $(4$ to $11 \mathrm{~cm}$ proximal to the plafond): plating versus intramedullary nailing. $\mathrm{J}$ Orthop Trauma. 2008; 22: 307-311. 